\begin{tabular}{|c|c|}
\hline Rinus Roelofs & Research \\
\hline $\begin{array}{r}\text { Lansinkweg } 28 \\
7553 \text { AL Hengelo } \\
\text { THE NETHERLANDS }\end{array}$ & $\begin{array}{l}\text { Two- and Three-Dimensional } \\
\text { Constructions Based on Leonardo Grids }\end{array}$ \\
\hline $\begin{array}{r}\text { rinus@rinusroelofs.nl } \\
\text { Keywords: Leonardo da } \\
\text { Vinci, grids, structural } \\
\text { patterns, tilings }\end{array}$ & $\begin{array}{l}\text { Abstract. In } 1989 \text { I made a drawing of a net on a cube, consisting of } \\
12 \text { lines/elements. They were connected in a way that, a couple of } \\
\text { months later, I recognised them in } 899 \mathrm{v} \text { in Leonardo's Codex } \\
\text { Atlanticus. I don't know which moment impressed me the most: my } \\
\text { own discovery of a very simple and powerful connecting system or } \\
\text { the discovery of the Leonardo drawings, which implied that my own } \\
\text { discovery was in fact a rediscovery. What we see in Leonardo's } \\
\text { drawings are some examples of roof constructions built with a lot of } \\
\text { straight elements. These drawings can be 'translated' into the } \\
\text { following definition: On each element we define four points at some } \\
\text { distance of each other - two points somewhere in the middle and } \\
\text { two points closer to the ends. To make constructions with these } \\
\text { elements we need only connect a middle point of one element to an } \\
\text { end point of another one in a regular over-under pattern. Out of the } \\
\text { simple definition of the elements, I designed many different patterns } \\
\text { for my so-called "+ - - +" structures: domes, spheres, cylinders and } \\
\text { other models were made. }\end{array}$ \\
\hline
\end{tabular}

\title{
Introduction
}

In 1989, I began constructing domes using notched bars assembled according to a simple rule. This led me to explore planar constructions based on this rule using fixed length "notched" linear segments. I was able to create a wide variety of patterns. From certain of these patterns I was able to construct spheres and cylinders with notched curved rods without the use of glue, rope, nails, or screws.

On folio 899v of the Codex Atlanticus we find, among others, three patterns with exactly the properties of these bar grids. In view of the way in which the patterns are drawn, oblong forms that seem to lie on each other, the most direct interpretation is that here we are dealing with stacking constructions, built from straight rods. Making a model leads to exactly the domes that I experimented myself. So the conjecture that Leonardo da Vinci is the first inventor of these constructions seems justified, although we cannot be sure about this.

Thus, the name I gave to my bar grid construction system is "Leonardo grids", with which I was able to construct all kinds of structures out of simple elements using one single constructing rule. Most of the constructions I made where planar and static. However, the "Leonardo grid system" also makes possible the construction of non-planar and dynamic structures.

\section{The system}

The construction admits a simple description. We start with a number of rods, on each one determining four points as indicated in fig. 1. We call these points connecting points.

Nexus Network Journal 10 (2008) 17-26

NEXUS NETWORK JOURNAL - VOL. 10, NO. 1, 200817

1590-5896/08/010017-10 DOI 10.1007/ S00004-007-0053-Y

(C) 2008 Kim Williams Books, Turin 
Fig. 1

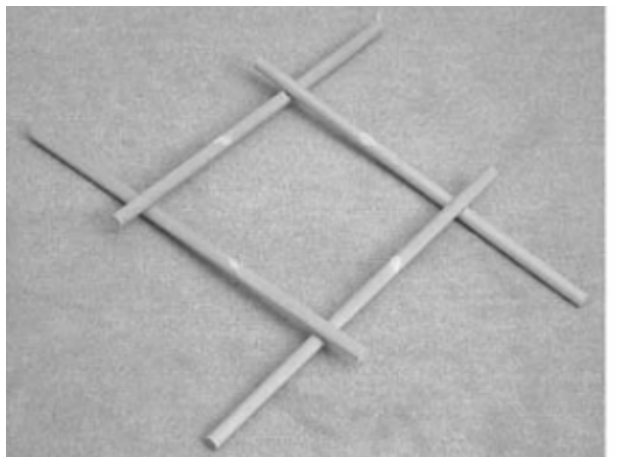

Fig. 2

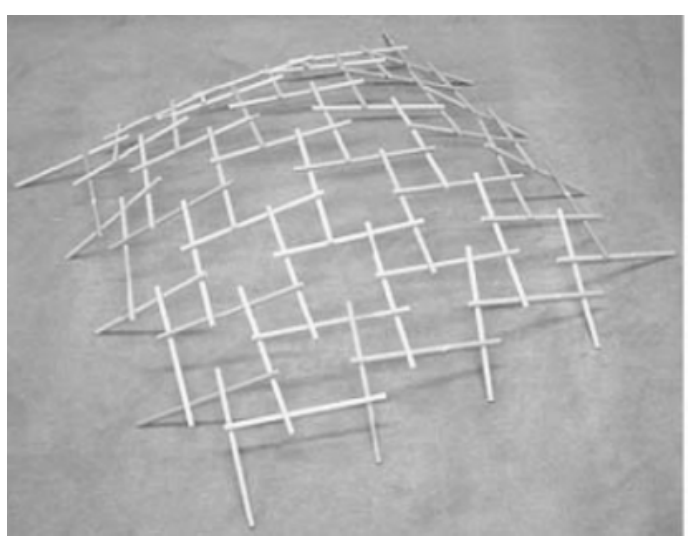

Fig. 4

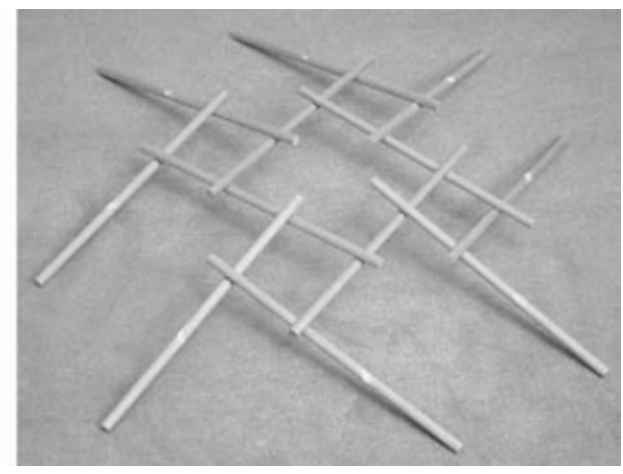

Fig. 3

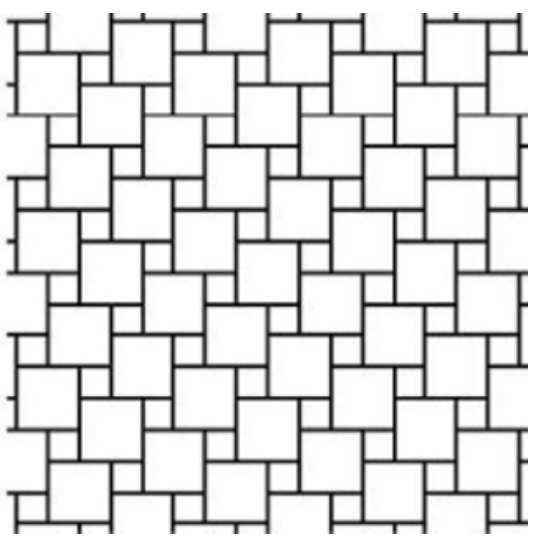

Fig. 5

We distinguish two types of connecting points: end points (closest to the ends of the rods) and interior points (the remaining points). Each rod has two end points and two interior points. In constructing the dome we now apply the following rules: one of the endpoints of a rod is placed on a free interior point of a different rod. At the end all connecting points of the rods have to be used as a connection between two rods, except near the border of the construction.

Now the actual construction of the dome turns out to be a simple task. Beginning with four rods, as in fig. 2, we extend the construction by continually adding rods at the bottom (fig. 3). Since we add one rod at the time, on the outer edge, the dome can be constructed by a single person. The four rods with which we have started will rise automatically during the building process and, at the end, the dome, consisting of 64 rods, will stand on the ground, resting on only 16 rods (fig. 4).

18 Rinus Roelofs - Two- and Three-Dimensional Constructions Based on Leonardo Grids 
Following the above construction process various patterns can be formed, each leading to a dome-like construction. In the sequel we will call the patterns that can be formed with the above rules "bar grids". The bar grid of the dome of fig. 4 can be drawn simplified as in fig. 5. In this form the drawing looks like a tiling pattern. However, we are not interested in the tiles but in the joints between the tiles. So we have a grid consisting of straight lines representing the rods. A first investigation into the various possible bar grids soon resulted into dozens of patterns, some of which are shown in fig. 6 .
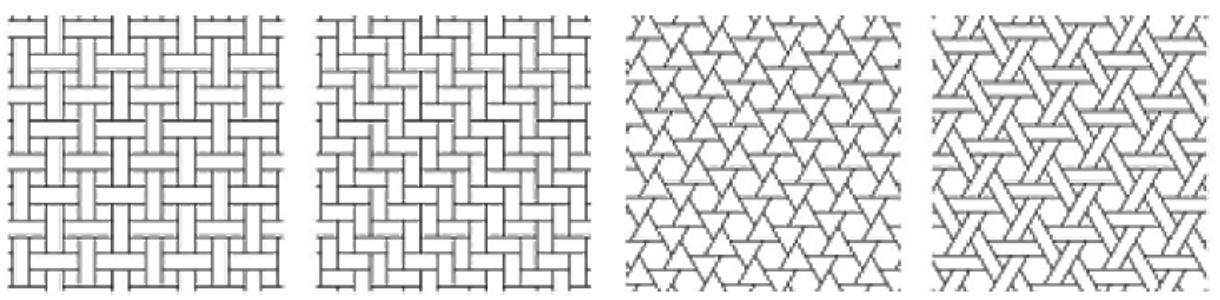

Fig. 6

\section{From $2 D$ to $3 D$}

In the domes it is gravity that keeps the loose rods together. It follows that continuing the construction as far as a complete sphere is not possible. Yet it turns out that, using the above construction system, objects can be formed in which the elements themselves, instead of gravity, keep the construction together. For example, we can assemble a sphere from a number of rods, or more generally elements, without using connecting materials like wire or glue. The number of connecting points per elements and the connecting rules do not change. It is only the form of the elements that changes. For a sphere we use curved rods instead of straight ones.

A simple way to come to a design for such a sphere shaped construction is the following: in the bar grid of fig. 7 the midpoints of the hexagons are connected such that a pattern of triangles results (fig. 8). Eight of these triangles can be used to form an octahedron (fig. 9).

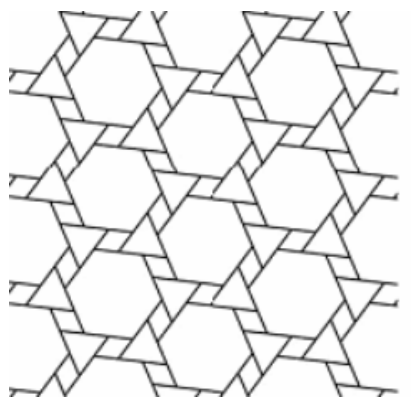

Fig. 7

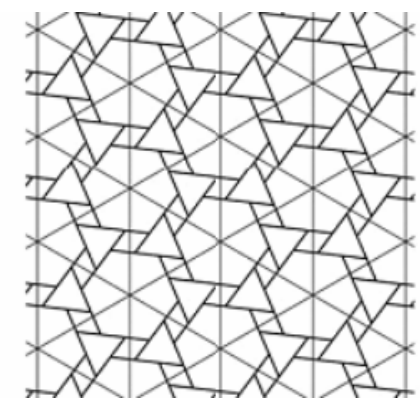

Fig. 8

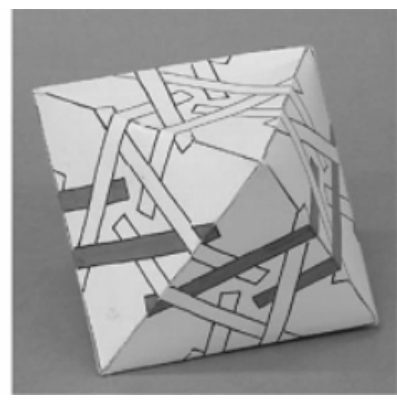

Fig. 9

On this octahedron we now see a grid consisting of 24 bars and this can be used as a design for the sphere of fig. 10. The form of the elements has been determined such that no tension arises in the sphere. Only when closing the sphere some elasticity from the elements is required. The relative position of the elements causes the sphere to stay in one piece: each 
of the elements is prevented from falling by other elements. For the sphere of fig. 11, which consists of 90 elements, the icosahedron has been used as an intermediate step so that pentagons occur in the construction.

Beside domes and spheres other shapes have been realized, such as cylinders and ovoids (figs. 12, 13).

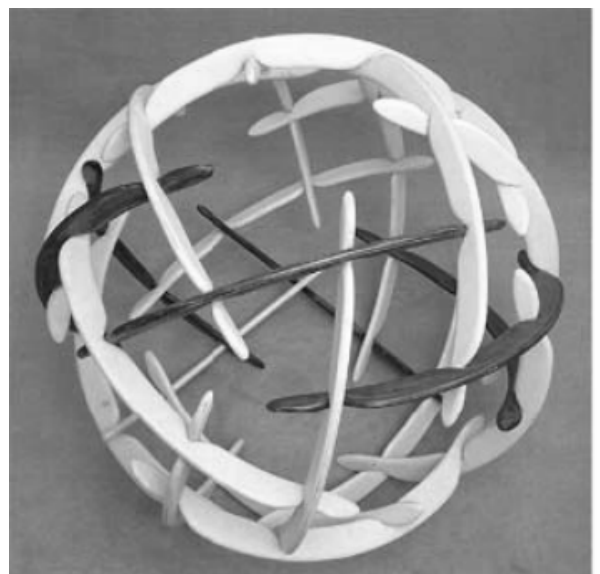

Fig. 10. Sphere, 24 elements sphere

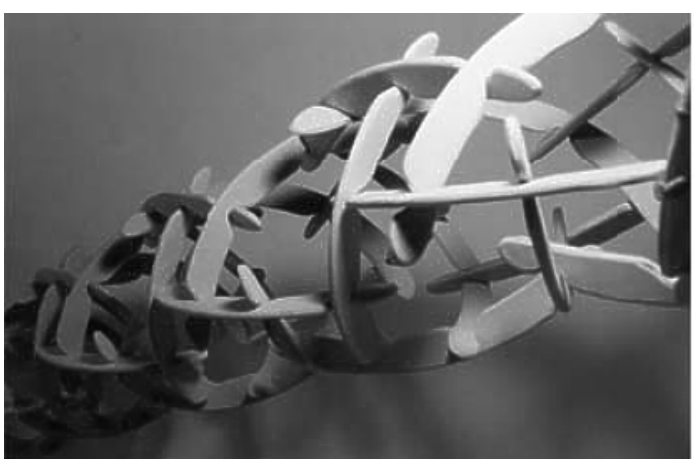

Fig. 12. Cylinder

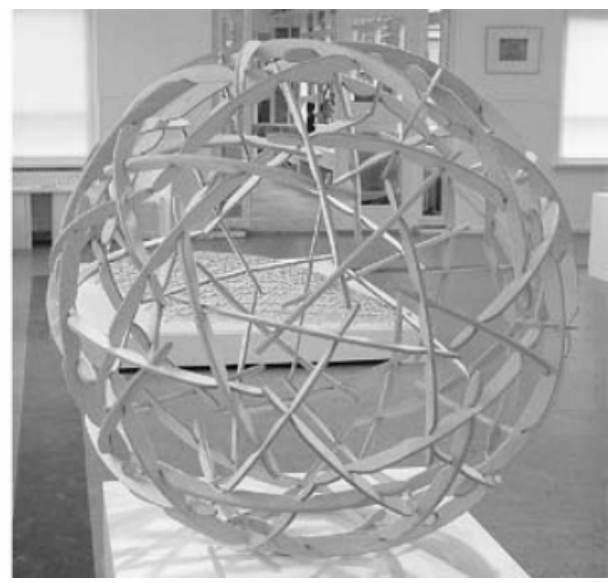

Fig. 11. Sphere, 90 elements

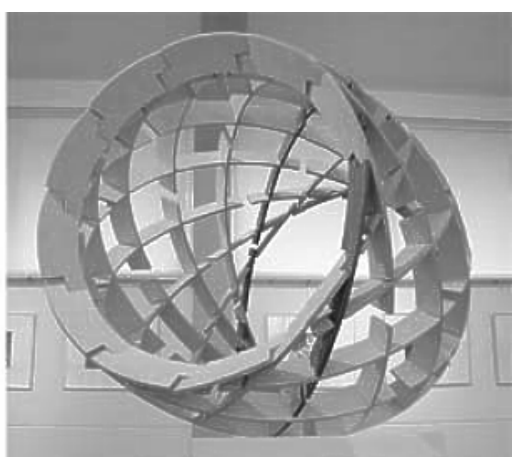

Fig. 13. Ovoid

A real new step was made when designing some objects in which the inner space of the sphere is used too, as in fig. 17. This object has the form of two linked concentric spheres. The whole is a stable construction consisting of 24 elements. Each element is halfway (that is to say with two out of four connecting points) in the outer sphere and halfway in the inner sphere. The design was made by starting with two layers of Leonardo grids. The layers were placed above each other in such a way that after cutting each element in two parts, half elements from the upper layer could be connected to half elements of the under layer. The resulting structure again has all the properties needed for a Leonardo grid: each element is connected to 4 other elements in the right way: all endpoints connected to midpoints and all midpoints connected to endpoints. Half of the connecting points are on the surface of the inner sphere, the other half on the surface of the outer sphere. This is the

20 RINUS ROELOFS - Two- and Three-Dimensional Constructions Based on Leonardo Grids 
first non-planar or real 3D result. And surprisingly enough, the total structure appeared to be stable.

Another approach was to start with interwoven patterns (fig. 14). A simple way to transform a flat pattern into a spherical construction is the use of polyhedron. When the pattern is hexagonal, the net of a icosahedron can be used. In the special case of the three interwoven patterns of fig. 15, the cut-off elements of pattern A will be connected to the cut-off elements of pattern $\mathrm{B}$ when folding the net into an icosahedron. And so with the cut-off elements of $\mathrm{B}$ and $\mathrm{C}$ and of $\mathrm{C}$ and $\mathrm{A}$.

The result is real 3D Leonardo grid construction. And now all the connecting points are laying in the same spherical surface. In the pictures (figs. 16, 17, 18) you can see some variations.

Although this is a good approach it is hard to find a good set of interwoven patterns that can be used for this method.
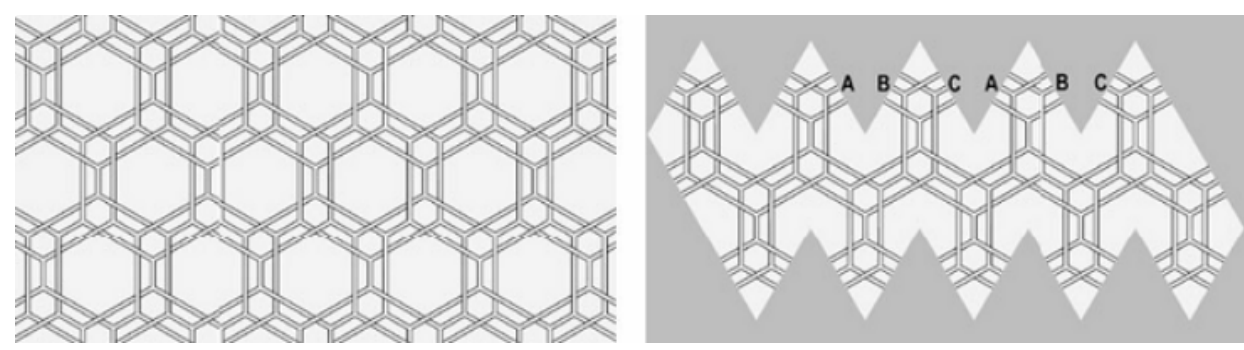

Fig. 14. Three interwoven patterns

Fig. 15. Icosahedron, plan
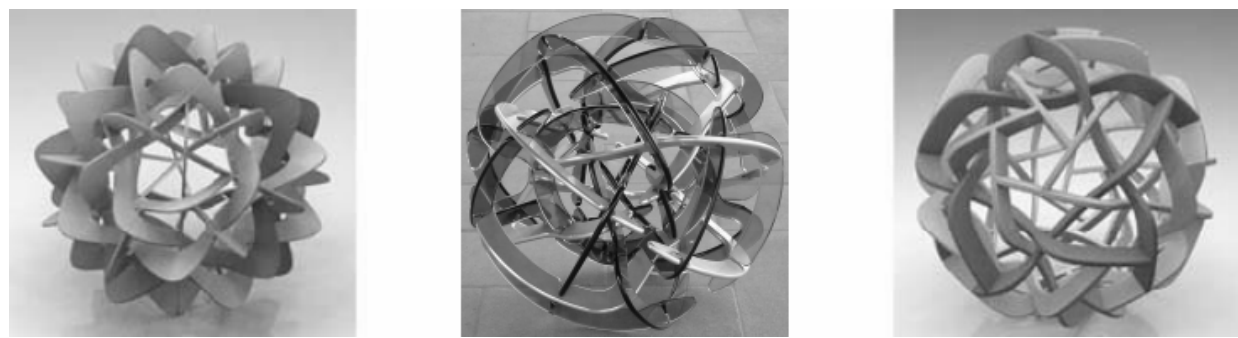

Figs. 16-18. Interwoven spheres

\section{Infinite double layer structures}

To go one step further towards the Leonardo grid space frames, I first tried to find a way to construct infinite double layer structures. Space frames can be built by connecting polyhedra in systematic way. With cubes you can fill the space. And when you look at the graph that represents the cube you will notice that all vertices have degree 3, which was a condition for the Leonardo grids. A cubic frame can be made as a Leonardo grid construction in three different ways (figures 19, 20 and 21).

A way to make a double layer structure is to connect these kinds of cubes as in fig. 22. This will result in non-planar infinite construction that has also dynamic properties. The elements can slide between certain boundaries and the total construction can be pressed together or stretched (figs. 23, 24). 


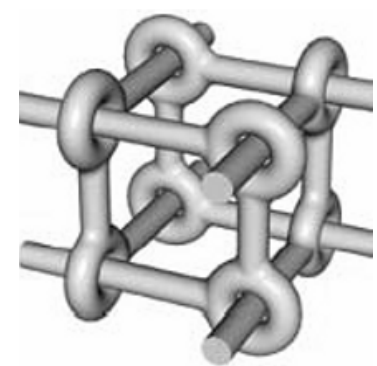

Fig. 19. Cubic frame A

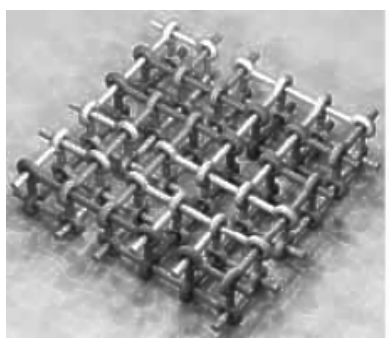

Fig. 22. Double layer structure

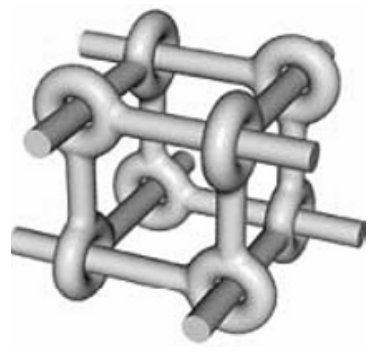

Fig. 20. Cubic frame B

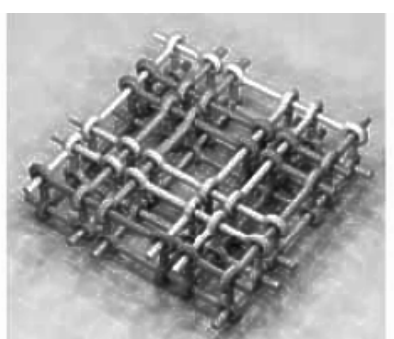

Fig. 23. Double layer structure, pressed together

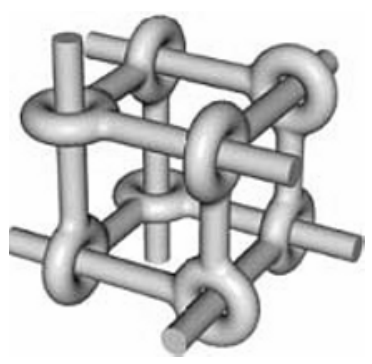

Fig. 21. Cubic frame C

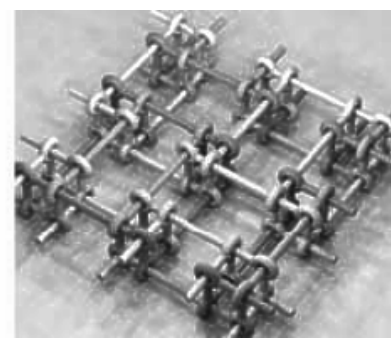

Fig. 24. Double layer structure, stretched

\section{Rings and strings}

In the double layer structures the basic elements, the rods with the 4 connecting points, are linked together to form bigger units. We can distinguish two kinds of these bigger units: rings, a closed concatenation of a finite number of basic elements, and strings a (open) concatenation of an infinite number of basic elements. Some examples of both categories are shown in figs. 25, 26 and 27 (rings) and figs. 28, 29 and 30 (strings).

The constructions needn't limited to two layers, as can be seen in figs. 31, 32 and 33. Here an infinite $3 \mathrm{D}$ construction is build with one type of string, which is a concatenation of basic Leonardo grid elements.

The question still is whether it is possible to make space frame constructions out of basic elements, which are not linked.

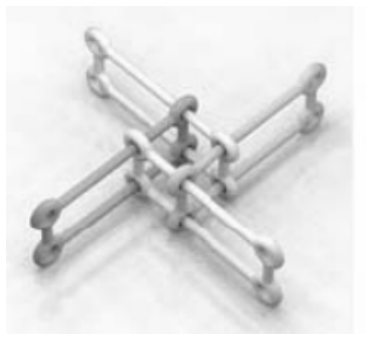

Fig. 25. Double layer structure

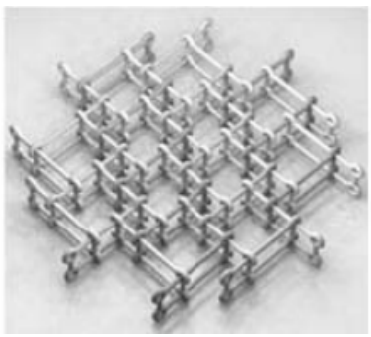

Fig. 26. Pressed together

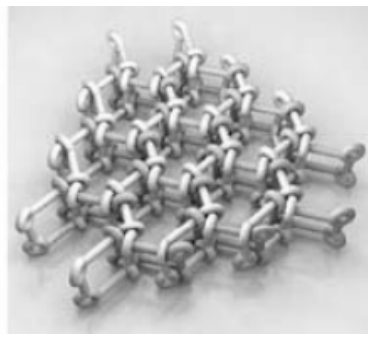

Fig. 27. Stretched

22 RINUS ROELOFS - Two- and Three-Dimensional Constructions Based on Leonardo Grids 


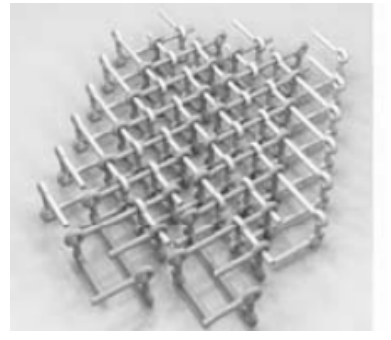

Fig. 28. Double layer structure

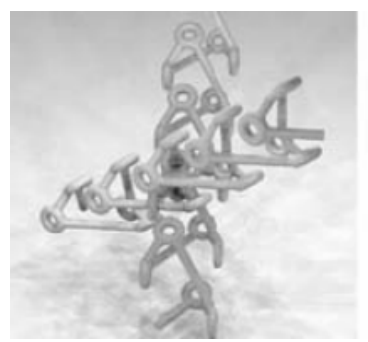

Fig. 31. 3D Strings

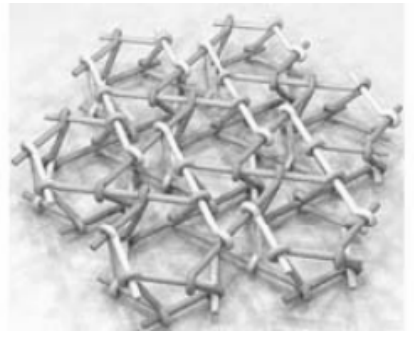

Fig. 29. Pressed together

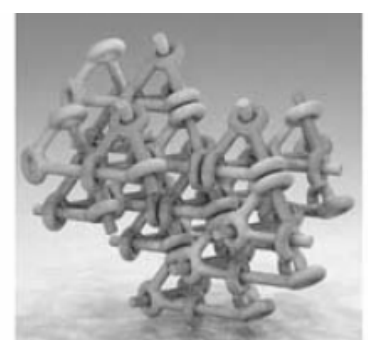

Fig. 32. 3D String structure

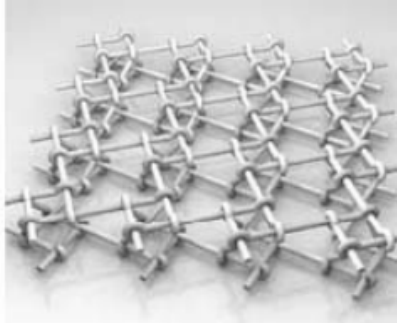

Fig. 30. Stretched

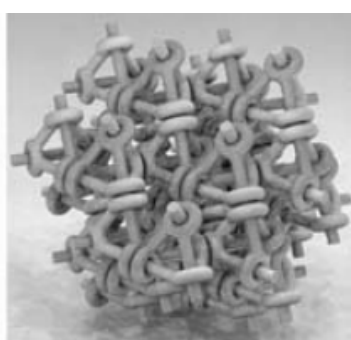

Fig. 33. 3D String structure

\section{Grid transformation}

Another, and maybe even better way to construct real 3D structures based on Leonardo grids appeared to be the use of transformation of the basic Leonardo grid from $2 \mathrm{D}$ to $3 \mathrm{D}$ (figs. 34, 35). The process can be described as follows: we can start with any pattern in which we have a hexagonal hole. We now keep the 6 sticks around this hole connected and change the hexagon from flat to skew. This change will cause a transformation of the sticks, which are connected to the first 6 sticks. The six parallelogram shaped holes in the pattern will also be parallelogram-shaped at the end of the process. But one of the connections around the triangular holes will get loose. The resulting structure now can be used as a layer with which we can create space frames.

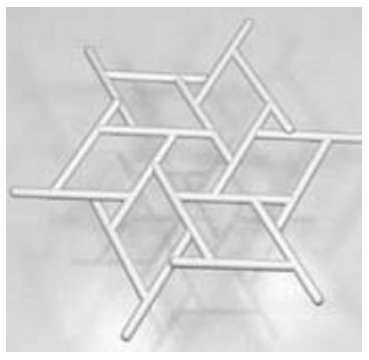

Fig. 34. Leonardo grid 2D

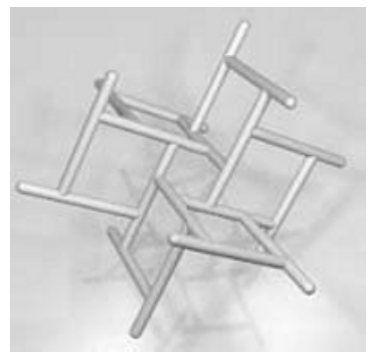

Fig. 35. Leonardo grid 3D

The discovery of this process lead to many designs of Leonardo grid space frames because the process could be applied on all the flat basic patterns. We can also start with the pattern with a square hole. In the same way the flat hexagon is transformed into a skew hexagon, a flat square can be transformed into a skew square. The over a hundred different 
patterns that I have drawn as possible designs for domes can now be transformed into Leonardo space frames. Fig. 36 shows one example of such space frames.

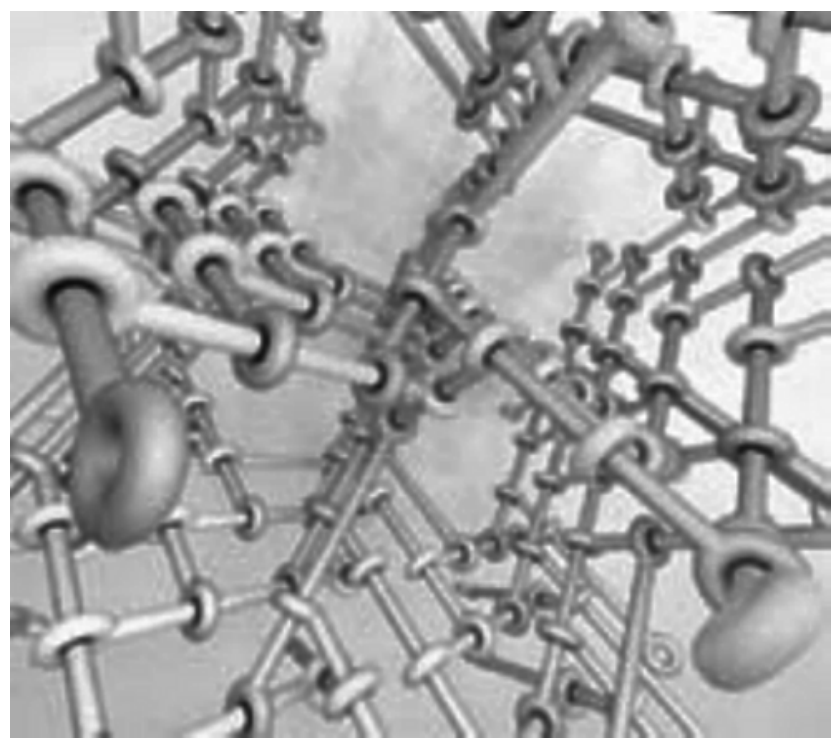

Fig. 36. Space frame grid

\section{Dynamic space frames}

Like the double layer structures, the Leonardo grid space frames also have dynamic properties. The sticks can be slid along each other and so the total construction can be pressed together or stretched. To show this we will go back to the skew hexagon first. In figs. 37, 38 and 39 you see three stages of the sliding process. And we can extend that to a complete layer, as in figs. 40, 41 and 42. In the total movement it looks as if there is some twist in the structure. The shrinking and growing of the structure is in a way a spiral movement. This can be best viewed in animation.
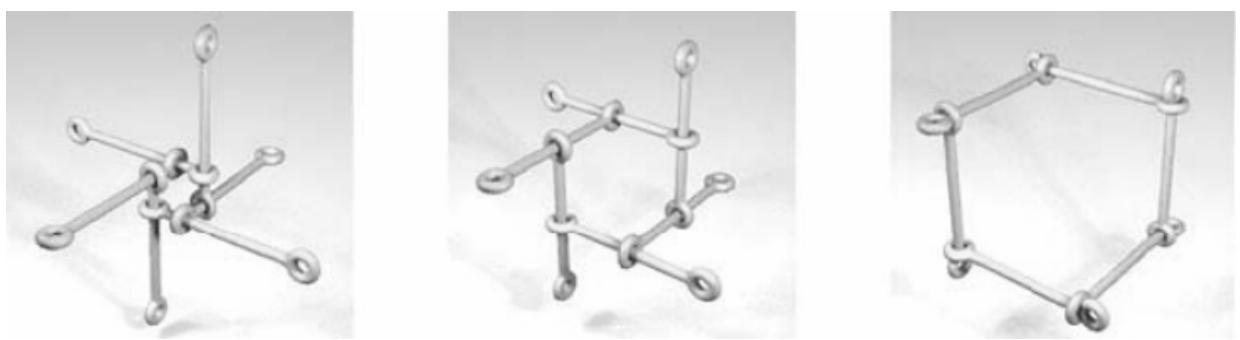

Figs. 37-39. Sliding: first, second, and third phases

24 RINUS ROELOFS - Two- and Three-Dimensional Constructions Based on Leonardo Grids 

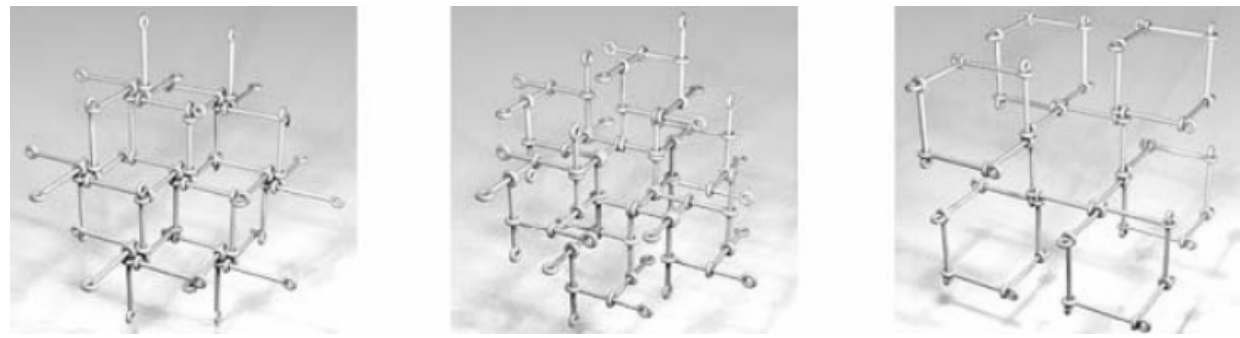

Figs. 40-42. Sliding: first, second, and third phases

\section{Dynamics in 2D}

While studying dynamics in the 3D Leonardo grid constructions I also discovered an interesting new way of translating 2D Leonardo grids into dynamic structures. Looking again at the basic grids, you have to realize that there are two possible interpretations: you can either look at it as a construction built out of rods, or as a tiling, a pattern built with a set of tiles. The dividing lines then form the Leonardo grid.
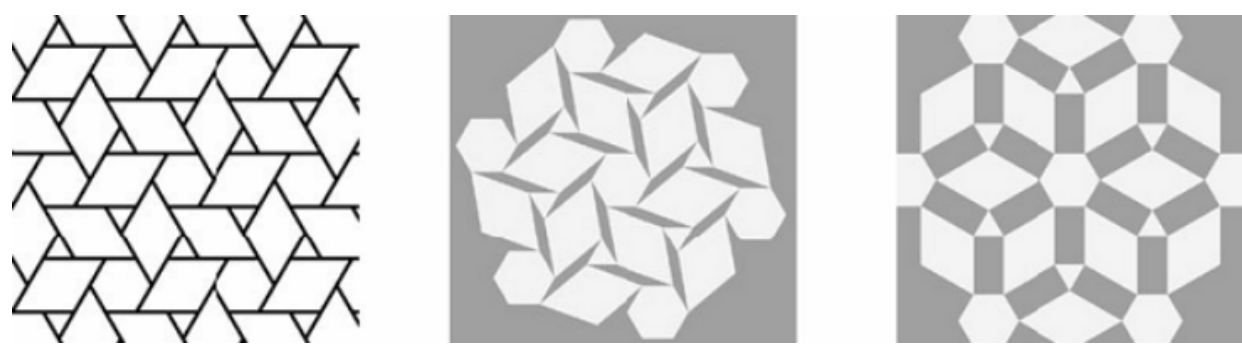

Figs. 43-45. Transforming: first, second, and third phases
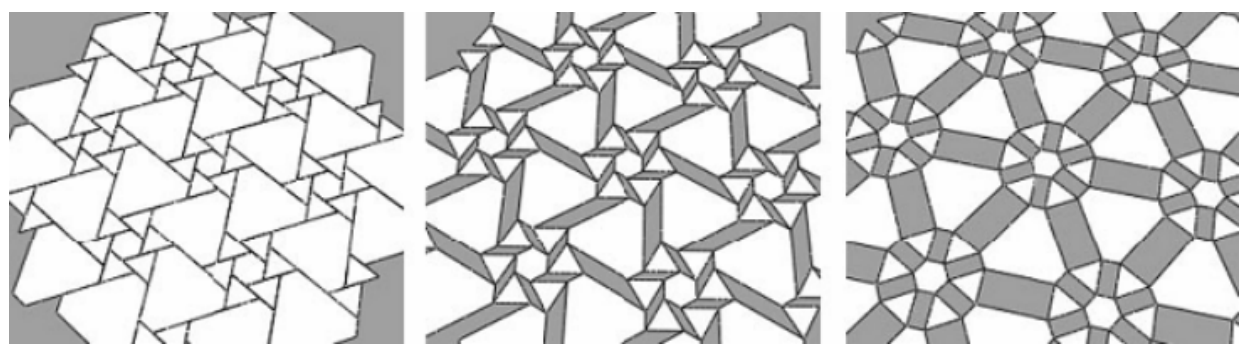

Figs. 46-48. Transforming: first, second, and third phases

One line in this grid represents the edges of 4 tiles: 2 big and 2 small tiles. So this one gridline can be seen as a set of 4 edges. Because the edges are alternately long-short-longshort, the set of edges can be seen as a parallelogram. In the original grid, the tiles are close to each other so the joints have area zero. But what will happen when we 'open' the parallelogram? The set of tiles then turns out to be a dynamic hinged construction. The Leonardo gridlines transform from lines to parallelograms to rectangles, and via parallelograms, to Leonardo gridlines again. And as a result of this process, a left-hand oriented Leonardo grid has been transformed into a right-hand oriented grid. 
Because this process can be applied on any Leonardo grid, this leads to an enormous collection of hinged tile constructions. Most special is that this process also works on Leonardo grids with distortions, that is, Leonardo grids in which more then one pattern is used, and on Leonardo grids in which more then one length of grid lines is used. Some examples can be seen in the figures.

Of course, this can be better viewed in animation.

\section{About the author}

Rinus Roelofs of Hengelo, Netherlands, is an artist. He was born in 1954. After studying Applied Mathematics at the Technical University of Enschede, he took a degree from the Enschede Art Academy with a specialization in sculpture. His commissions come largely from municipalities, institutions and companies in the Netherlands, but his work has been exhibited further afield, including in Rome as part of the Escher Centennial celebrations in 1998. More information about his activities is available on his website, http://www.rinusroelofs.nl.

26 RINUS ROELOFS - Two- and Three-Dimensional Constructions Based on Leonardo Grids 